\title{
Mid- to Long-Term Follow-up Outcomes of One-Stage Posterior Lumbosacral or Lumbopelvic Fixation in the Management of Lumbosacral Junction Tuberculosis in Adults
}

\section{Zhenchao Xu}

Xiangya Hospital Central South University

\section{Zhen Zhang}

Xiangya Hospital Central South University

\section{Gang Chen}

Xiangtan Central Hospital

\section{Xiyang Wang ( $\nabla$ wqlwqwxy@163.com )}

Xiangya Hospital Central South University Department of Spinal Surgery https://orcid.org/0000-0003-1942-7517

\section{Research article}

Keywords: lumbosacral region, orthopedic surgery, posterior approach, spinal tuberculosis, spondylodiscitis

Posted Date: November 2nd, 2020

DOl: https://doi.org/10.21203/rs.3.rs-99414/v1

License: (a) (1) This work is licensed under a Creative Commons Attribution 4.0 International License. Read Full License 


\section{Abstract}

Background: This retrospective study aimed to evaluate the mid- to long-term outcomes of surgical management of lumbosacral junction tuberculosis in adults.

Methods: A total of 38 adult patients were treated by one-stage posterior debridement, interbody fusion, lumbosacral or lumbopelvic fixation, and postural drainage. All cases were followed up for at least five years. Clinical and radiographic outcomes were gathered and analyzed.

Results: All patients achieved clinical cure at the last follow-up. Erythrocyte sedimentation rates decreased to normal levels within three months postoperatively. Postoperative scores measuring pain (visual analog scale), neurological status (Japanese Orthopedic Association score), and patient quality of life (Oswestry Disability Index) all significantly improved compared to preoperative values. Patient-reported outcomes as measured by Kirkaldy-Willis criteria were excellent in 21 cases, good in 16 cases, and fair in 1 case; there were no poor outcomes. Lumbosacral angle increased from the preoperative values of $21.7^{\circ} \pm 1.8^{\circ}$ to the postoperative values of $26.4^{\circ} \pm 1.4^{\circ}$, with an angle loss of $1.2^{\circ} \pm 0.7^{\circ}$ at the last follow-up. Bone fusion occurred on average $12.8 \pm 1.9$ months after surgery. No nonunion, pseudarthrosis, loosening or fracture of instruments occurred at the last follow-up.

Conclusion: For patients with lumbosacral junction TB, a treatment protocol including one-stage posterior debridement, interbody fusion, lumbosacral or lumbopelvic fixation, and postural drainage according to the severity of sacral destruction is a relatively nontraumatic and highly safe procedure. It can be used to reconstruct lumbosacral or lumbopelvic stability and facilitate the healing of TB.

\section{Background}

According to the Global Tuberculosis Report released by the World Health Organization in 2019, there were approximately 10 million new cases of tuberculosis (TB) worldwide in 2018, making it one of the top ten causes of death in the world [1]. The spinal column is the most common site of infection for extrapulmonary TB, accounting for approximately $50 \%$ of osteoarticular TB [2]. This severe spinal disease often causes spinal instability, kyphotic deformity, neurological dysfunction, and even paraplegia [3]. Spinal TB involving the lumbosacral junction is rare, and only accounts for 2-3\% of the total number of spinal TB cases reported in the literature [4]. Conservative treatment with anti-TB drugs is the primary approach for the condition [5]. However, simple drug therapy is often insufficient as the disease progresses and patients show complications with vertebral collapse and severe deformity, which may lead to lumbosacral malunion and chronic lower back pain. Hence, surgeons are increasingly adopting chemotherapy with surgery to solve this dilemma [6].

Various surgical methods including an anterior approach, a combined anteroposterior approach and a posterior approach have been described for treating lumbosacral junction TB [7-9]. Surgery promotes the quiescence of the abscessed area by lesion clearance, bone grafting and internal fixation, so that the infection can be clinically eliminated [10]. However, as the transition zone between the lumbar vertebrae and the sacrum, the unique anatomical structure and biomechanical characteristics of the lumbosacral segment bring challenges to the operation. The adjacent organs in the lumbosacral area not only need to be protected from damage, but one also needs to pay special attention to the reconstruction of lumbosacral stability after lesion removal [11]. Due to a lack of evidence and guidelines regarding optimal treatment and management strategies, the treatment of lumbosacral junction TB remains a difficult and challenging decision-making process. Currently, reports on the management of lumbosacral junction TB are relatively scarce due to its low incidence rate.

In this study, 38 patients with lumbosacral junction TB who underwent one-stage posterior lumbosacral fixation or lumbopelvic fixation, and postural drainage were followed up for more than five years. We evaluated the mid- to long-term 
clinical outcomes and effectiveness of surgical management in helping these patients eliminate the infection, regain lumbosacral or lumbopelvic stability, resolve neurological impairments and restore normal quality of life.

\section{Methods}

\section{Patient diagnosis, inclusion and exclusion criteria}

A total of 38 adult patients with lumbosacral junction TB were enrolled in this study from February 2008 to July 2015. Written informed consent was obtained from all patients prior to the study, and the ethics committee of Xiangya hospital approved the study protocol. The primary diagnosis of tuberculous spondylodiscitis or spondylitis was made on the basis of clinical, neurological, hematologic, imaging and laboratory data. Final confirmation of diagnosis was achieved through histopathologic analysis of a biopsied sample or a positive tubercle bacillus culture.

The inclusion criteria for surgery were the following: (1) persistent lower back pain caused by instability; (2) severe or progressive neurological impairment; (3) unpreventable progressive deformity; or (4) weak efficacy of anti-TB drugs, as confirmed by drug sensitivity testing. The exclusion criteria were individuals who had (1) already undergone lumbosacral surgery and thus showed unclear morphology in the area, (2) had history of congenital scoliosis or ankylosing spondylitis, or (3) showed multi-level lumbosacral junction TB (exceeding two segments that required surgical treatment) with iliac fossa or anterior abscess formation. A total of 38 patients were included and followed for at least five years with complete data.

\section{Preoperative procedure}

Anti-TB treatment with the HREZ chemotherapy regimen was routinely carried out for at least two weeks prior to surgery. The dosages of each drug were the following: isoniazid ( $\mathrm{H} ; 5 \mathrm{mg} / \mathrm{kg} / \mathrm{day},<300 \mathrm{mg} /$ day), rifampicin $(\mathrm{R} ; 10 \mathrm{mg} / \mathrm{kg} / \mathrm{day},<$ $1200 \mathrm{mg} /$ day), ethambutol (E; $15 \mathrm{mg} / \mathrm{kg} /$ day, < $2500 \mathrm{mg} /$ day), and pyrazinamide (Z; $30 \mathrm{mg} / \mathrm{kg} /$ day, $<2000 \mathrm{mg} /$ day). Nutritional support therapy was administered to correct hypoproteinemia and anemia. Surgery was postponed until erythrocyte sedimentation rate (ESR) values, which indicated the activity level of the TB infection, either decreased significantly or returned to normal. If a patient experienced cauda equina syndrome or dramatic aggravation of neurological impairment during the anti-TB treatment, emergency surgery was performed regardless of the ESR value.

\section{Surgical methods}

The key surgical procedure included mounting pedicle screws on one or two segments above the infected vertebra through a posterior median incision. Pedicle screws were inserted in the S1 vertebra in cases where the vertebral body was slightly damaged so that the integrity of the pedicles was preserved. If the $S 1$ vertebra and pedicle channel were severely damaged, iliac screws were fixed to the iliac wings. A hemi-laminectomy or laminectomy was performed on the severely damaged side of the lesion segment. The superior and inferior articular processes of the pathologic vertebrae were partially resected to expose the affected vertebral bodies. Curettes of varying angles were used to remove the lesion, including sequestrum, necrotic intervertebral disc and tuberculous granuloma. Pus and necrotic tissue were removed by negative pressure irrigation and negative pressure suction via a soft catheter, which was placed deep into the lesion. The same procedure was performed on the other side, if necessary. The bony surfaces of the vertebral body were repaired by bone grafts. Installation of permanently contoured rods and compression with a cantilever bending technique under vision were employed to achieve local spinal alignment. A suitable allogeneic bone block was implanted between the vertebrae to complete the reconstruction of the anterior middle column. Moreover, autologous and allogeneic particulate bone was implanted between the bilateral transverse processes. Streptomycin $(0.1 \mathrm{~g})$ combined with isoniazid $(0.3 \mathrm{~g})$ were used locally in the focal area, and the incision was closed in layers after a drainage tube was placed.

Histopathologic examinations and Mycobacterium cultures were performed on the surgical specimens of each patient. 


\section{Postoperative procedure}

Typically, the drainage tube was removed once the volume of drainage became less than $30 \mathrm{~mL}$ within 24 hours. All patients continued with the HREZ chemotherapy regimen for 12-18 months after surgery. Intravenous antibiotics and nutritional support were administered to prevent infection. Rehabilitation training and physical therapy were provided to all patients as early as possible to help them regain neurological function and prevent thrombosis. With the assistance of a rigid lumbosacral brace, the patients were permitted to walk gradually after strict bed rest for four weeks post-surgery. The external brace was removed when imaging data from the patients revealed formation of a callus. Routine blood and hepatorenal function tests, as well as ESR tests, were performed to monitor the side effects and to evaluate the clinical efficacy of the treatment. Regular re-examinations through outpatient services were conducted every three months in the first year postoperatively, and every six months thereafter. At least five years of follow-up were carried out for each patient to obtain clinical and radiologic data.

\section{Evaluation standards}

ESR was measured to indicate the activity level of the TB infection perioperatively and postoperatively. Imaging approaches including radiography, computed tomography (CT), and magnetic resonance imaging (MRI) were used to detect narrowing of the intervertebral space, vertebral body collapse, spinal instability, bone destruction and paravertebral and epidural abscess formation during diagnosis and preoperatively. Routine radiography and CT scans were also carried out postoperatively and at follow-up to assess the placement of instruments and extent of decompression, as well as to classify bone fusion based on the modified radiologic criteria by Lee et al. [12]. The visual analog scale (VAS) score was used to assess pain intensity. The Japanese Orthopedic Association (JOA) score [13] and Oswestry Disability Index (ODI) [14] were used to evaluate neurological function and quality of life in patients, respectively. VAS, JOA and ODI together with lumbosacral angle were measured preoperatively, postoperatively, and at the last follow-up. Finally, the Kirkaldy-Willis criteria [15] was used to determine the functional outcome at the end of the observation period for each patient.

\section{Statistical analysis}

Statistical analyses were performed using SPSS version 20.0 (IBM Corp., Armonk, NY). The clinical and radiographic data collected preoperatively, postoperatively and at the final follow-up were compared using a paired t-test. Discrepancies of the normal distribution were evaluated using the rank sum test. A p value $<0.05$ was considered statistically significant.

\section{Results}

The patient demographic data and disease characteristics are detailed in Table 1. Among the patients who met the inclusion criteria, 24 were male and 14 were female. The mean age at time of surgery was $48.0 \pm 13.0$ years. All cases presented with TB symptoms such as low back pain, weakness, weight loss and low fever. In addition, 18 cases (47.4\%) had radicular pain, 9 cases (23.7\%) showed lower limb dysfunction, and 1 patient (2.6\%) suffered from cauda equina syndrome. The ESR values were found to be raised at the initial stage before surgery. Histopathologic examinations of surgical specimens from all 38 patients confirmed the presence of tuberculous granuloma or caseous necrosis. Of these, 11 cases showed positive culture for Mycobacterium tuberculosis. The average length of the postoperative follow-up was $66.2 \pm 4.4$ months; all patients had completely resolved their infections at the final follow-up. 
Table 1

Patient demographic, disease characteristics and operative information.

\begin{tabular}{|c|c|c|c|c|c|c|}
\hline \multirow{2}{*}{$\begin{array}{l}\text { Total } \\
\text { patients (n) }\end{array}$} & \multicolumn{2}{|c|}{ Gender } & \multirow{2}{*}{$\begin{array}{l}\text { Age } \\
\text { (years) }\end{array}$} & \multirow{2}{*}{$\begin{array}{l}\text { Duration of symptoms } \\
\text { (months) }\end{array}$} & \multirow{2}{*}{$\begin{array}{l}\text { Intraoperative blood } \\
\text { loss }(\mathrm{mL})\end{array}$} & \multirow{2}{*}{$\begin{array}{l}\text { Operative } \\
\text { time (min) }\end{array}$} \\
\hline & $\begin{array}{l}\text { Male } \\
(n)\end{array}$ & $\begin{array}{l}\text { Female } \\
\text { (n) }\end{array}$ & & & & \\
\hline 38 & 24 & 14 & $\begin{array}{l}48.0 \pm \\
13.0\end{array}$ & $4.7 \pm 2.5$ & $726.3 \pm 151.9$ & $137.6 \pm 22.5$ \\
\hline
\end{tabular}

The average intraoperative bleeding amount was $726.3 \pm 151.9 \mathrm{~mL}$, and the average operative time was $137.6 \pm 22.5$ minutes (Table 1). The ESR values were $67.9 \pm 21.9 \mathrm{~mm} / \mathrm{h}$ preoperatively, which significantly decreased to $11.8 \pm 2.6 \mathrm{~mm} / \mathrm{h}$ at three months post-surgery $(\mathrm{p}<0.05)$.

The VAS score significantly decreased from an average of $6.8 \pm 1.1$ preoperatively to $0.8 \pm 0.7$ at the final follow-up ( $p<$ 0.05). Similarly, scores for neurological function (JOA) and quality of life (ODI) at the last visit showed significant improvement when compared with preoperative scores $(p<0.05)$. At the end of the follow-up period, patients' functional outcome was assessed using the Kirkaldy-Willis criteria; 21 cases reported excellent results, 16 cases reported good results, and 1 case reported fair result. There were no reports of poor outcomes (Table 2).

Table 2

Clinical evaluation indices for preoperative and postoperative follow-ups.

\begin{tabular}{|c|c|c|c|c|c|c|c|c|c|c|c|c|}
\hline \multicolumn{3}{|c|}{$\mathrm{ESR}(\mathrm{mm} / \mathrm{h})$} & \multicolumn{2}{|l|}{ JOA } & \multicolumn{2}{|l|}{ VAS } & \multicolumn{2}{|l|}{ ODI } & \multicolumn{4}{|c|}{ Kirkaldy-Willis criteria } \\
\hline Pre & TMP & FFU & Pre & FFU & Pre & FFU & Pre & FFU & Excellent & Good & Fair & Poor \\
\hline $\begin{array}{l}67.9 \\
\pm \\
21.9\end{array}$ & $\begin{array}{l}11.8 \\
\pm \\
2.6^{*}\end{array}$ & $\begin{array}{l}7.6 \\
\pm \\
2.1^{*}\end{array}$ & $\begin{array}{r}18.5 \\
\pm 2.9\end{array}$ & $\begin{array}{l}26.9 \\
\pm \\
1.1^{*}\end{array}$ & $\begin{array}{l}7.2 \\
\pm \\
0.9\end{array}$ & $\begin{array}{l}0.8 \\
\pm \\
0.7^{\star}\end{array}$ & $\begin{array}{l}44.3 \\
\pm 6.7\end{array}$ & $\begin{array}{l}9.3 \\
\pm \\
1.9^{*}\end{array}$ & 21 & 16 & 1 & 0 \\
\hline
\end{tabular}

* Analyzed by paired t test, compared with preoperative value, $p<0.05$. Data expressed as mean \pm standard deviation.

Abbreviations: Pre, preoperative; TMP, three months postoperative; FFU, final follow-up; ESR, erythrocyte sedimentation rate; JOA, Japanese Orthopedic Association score; VAS, visual analog scale; ODI, Oswestry Disability Index.

The lumbosacral angle significantly increased from the preoperative average of $21.7^{\circ} \pm 1.8^{\circ}$ to an average of $26.4^{\circ} \pm 1.4^{\circ}$, postoperatively. At the last visit, the lumbosacral angle was an average of $32.8^{\circ} \pm 3.6^{\circ}$, with a correction loss of only $1.2^{\circ} \pm$ $0.7^{\circ}$. There was no statistically significant difference between the lumbosacral angle measurements made immediately post-surgery and at the last follow-up. Spontaneous intervertebral bone fusion was achieved at $12.8 \pm 1.9$ months after surgery (Table 3). Nonunion, pseudoarthrosis, loosening or fracture of instruments had not occurred at the time of the final follow-up (Figs. 1 and 2). 
Table 3

Radiographic outcomes of preoperative and postoperative follow-ups.

\begin{tabular}{|llllll|}
\hline \multicolumn{4}{|l}{ Lumbosacral angle } & \multicolumn{3}{l}{ Correction rate (\%) } & & Fusion time (months) \\
\hline Pre & Post & FFU & Lost & & \\
\hline $21.7^{\circ} \pm 1.8^{\circ}$ & $26.4^{\circ} \pm 1.4^{\circ *}$ & $25.3^{\circ} \pm 1.3^{\circ *}$ & $1.2^{\circ} \pm 0.7^{\circ}$ & $22.3 \pm 6.2$ & $12.8 \pm 1.9$ \\
\hline
\end{tabular}

${ }^{*}$ Analyzed by paired t-test, compared with preoperative values, $p<0.05$. Data expressed as mean \pm standard deviation.

Abbreviations: Pre, preoperative; Post, postoperative; andFFU, final follow up.

None of the patients died or suffered permanent neurological damage. Two cases experienced superficial incisional infection, which was successfully controlled with antibiotics. Anti-TB drug-induced liver dysfunction occurred in a single patient, and was subsequently managed using modified chemotherapy combined with hepatic protection treatment.

\section{Discussion}

The lumbosacral spine is at the junction of a lordotic motion segment and a kyphotic fixed end, an area where the stress of the entire torso is concentrated. The intact facet joints and intervertebral discs of the lumbosacral segment are the main structures that resist the shear force of the L5 vertebral body forward. Hence, spondylolisthesis of the lumbosacral region can easily bring about force imbalance, such that firm internal fixations are usually required. Lumbosacral TB is relatively rare, and has an insidious onset and atypical symptoms. Individuals with the condition often report nonspecific symptoms, which are easily mistaken for spinal degenerative disease at the initial stage [16]. As the disease progresses, $M$. tuberculosis erodes the vertebrae to the point of sequestrum and abscess formation, leading to spinal instability or deformity. Additionally, nerve compression from TB lesions that invades the spinal canal potentially cause radicular pain, and even acute paralysis, which has deleterious effects on the patient's overall quality of life [17].

Standard systemic anti-TB chemotherapy remains the basic strategy throughout the entire treatment period $[18,19]$. In our study, normative anti-TB treatments were carried out on all patients for two weeks preoperatively and for 12-18 months postoperatively. Common indications for surgery in lumbosacral junction TB are ineffective conservative measures, persistent pain unrelieved by chemotherapy, progressive neurological deficits, relatively massive cold abscesses, and extensive vertebrae destruction with instability or deformity. The surgery is specifically carried out with the goal of removing the lesion, relieving nerve compression and rebuilding spinal stability, all of which aid the maintenance of normal lumbar lordosis and sacral kyphosis. This also ensures that the normal physiological line of force transduction is preserved. For patients who require surgery, the key techniques for surgical treatment of spinal TB include complete debridement, adequate bone grafting and firm internal fixation.

The surgical method for the treatment of TB in the lumbosacral junction is still controversial [20-22]. An anterior approach has been conventionally preferred since most TB lesions involve the vertebral body and intervertebral discs. The anterior approach allows for direct access where thorough debridement of the affected tissue and intervertebral reconstruction can be performed $[23,24]$. Nevertheless, the anterior approach is associated with anteriorly related complications. In particular, the lumbosacral region is an area with complex local anatomical structures and is adjacent to numerous vital organs, including major blood vessels, nerve plexi, and ureters [22]. Tribus et al. [25] reported that the distance from the bifurcation of iliac vessels to the top of the L5-S1 disc averaged $18 \mathrm{~mm}$, and the average space available between the left common iliac vein and the right common iliac artery is $33.5 \mathrm{~mm}$, a result they surmised from studying 37 human cadavers. This means that it is too risky to perform the surgery via the anterior approach, due to the close proximity of the vasculatures that this approach exposes. In addition, anterior instruments may be inadequate since the presence of the TB infection is associated with osteoporosis that renders the vertebrae increasingly fragile, thereby potentially causing graft failure and loss of correction after surgery [26]. These existing objective factors pose significant challenges to anterior fixation of the 
lumbosacral segment, while carrying the risk of damage to major neurovascular structures. Some surgeons report that anterior debridement combined with posterior instrumentation in the management of lumbosacral TB can obtain favorable clinical efficacy $[27,28]$. This approach overcomes the stability-related shortcoming when the anterior approach is used alone and encompasses a broader surgical vision and higher bone graft fusion rate. However, it also results in superimposed surgical trauma and increased complications. Taking the above factors into account, we favor the posterior approach for treating lumbosacral junction TB when surgery is indicated.

With the widespread application of pedicle screw-rod internal fixation systems, the satisfactory safety and clinical efficacy of the posterior-only approach for the treatment of lumbosacral TB has increasingly attracted the attention of orthopedic surgeons for many reasons $[7,17,26,29,30]$. Firstly, the posterior approach is less invasive and avoids damage to vital nerves and blood vessels. In addition, pedicle screws provide better holding force, and are thus superior to the anterior approach in correcting severe kyphosis. Zeng et al. [7] successfully treated 15 cases of lumbosacral TB with paraspinal abscess using the posterior approach. Similarly, Xu et al. [17] also reported that kyphotic deformity significantly improved with complete recovery of neurological function by interbody fusion and posterior lumbopelvic fixation for lumbosacral TB. In this study, all 38 cases showed complete resolution from TB infection by the last follow-up. The patients who suffered preoperative neurological deficit had satisfactory recovery at the final follow-up. In terms of spinal stability, over the five years of the observation period, patients presented an average loss of the correction angle of only $1.2^{\circ} \pm 0.7^{\circ}$ with a $22.3 \%$ $\pm 6.2 \%$ correction rate after surgery. Pain intensity as measured by VAS dropped by over $80 \%$, while at the last follow-up, 21 patients (55.3\%) reported excellent status, and 16 patients (42.1\%) reported good status based on Kirkaldy-Willis criteria.

The surgical treatment of lumbosacral TB should follow an individualized protocol. A routine CT scan and MRI examination are essential before surgery to map out the details of the lesion. When the $S 1$ vertebral body is severely damaged, the use of sacral pedicle screws cannot obtain sufficient bony support. The physiological force transmission line in the lumbosacral region is subsequently affected, which brings challenges to surgical reconstruction. The sacrum forms the posterior wall of the pelvis and is connected to the ilium through the sacroiliac joints. Physiologically, gravitational force is transmitted from the lumbosacral joint to the upper part of the sacrum, and then to the pelvis through the bilateral sacroiliac joints. Due to the special role it plays in pressure bearing and force transduction, the sacrum is considered the keystone of the pelvic ring [31]. For cases when S1 is severely damaged, lumbopelvic fixation can effectively relieve the stress on the diseased vertebra and promote its healing. In addition, iliac screw fixation can immediately stabilize the lesion and restore the normal lumbosacral angle [32]. For patients with an S1 vertebral body lesion that is not serious and with an intact pedicle channel, sacral pedicle screw fixation can effectively reconstruct local stability and cross spinopelvic fixation can be avoided.

M. tuberculosis tends to infect the anterior and middle column of the spine. The controversy with regard to surgical treatment of lumbosacral TB with the posterior approach mainly centers around whether this approach can allow for complete debridement given its limited field of view. Indeed, the posterior approach offers no advantage in lesion clearance. Nonetheless, resection of both sides of the lamina and facet joints, and moderate stretching of the dura mater and nerve roots can provide enough operating space for the removal of the lesion. Then, through intraoperative pressure washing, negative pressure suction and postoperative postural drainage, the sequestrum, necrotic tissue and most abscesses can be removed. The remaining small areas of infection can be resolved by standardized anti-TB chemotherapy after surgery [33]. Some surgeons are concerned that this surgical method destroys the posterior column and may cause spinal instability. However, pedicle screws, which allow for three-column fixation, can effectively restore the normal physiological curvature of the spine, and overcome the instability caused by column damage within a short time period. Sufficient intervertebral and transverse bone grafting provide long-term bone support for spine stability [34]. During the postoperative follow-up of this study, there were no cases of TB recurrence from incomplete lesion removal. In terms of structural recovery, all patients achieved satisfactory bone fusion at an average of $12.8 \pm 1.9$ months after surgery without a single incidence of instrument failure. 
This study has some limitations. Firstly, this is a retrospective study, which may lead to biased results. Furthermore, this study is a single-center study with a relatively small sample size. Prospective studies with larger sample sizes are required to confirm the findings, although given the rarity of this condition, it may take considerable time and coordination for such studies to take place.

\section{Conclusion}

For patients with lumbosacral junction TB where anti-TB chemotherapy is ineffective, or with worsening neurological deficits and pain, one-stage posterior debridement, interbody fusion, lumbosacral or lumbopelvic fixation, and postural drainage, according to the severity of sacral destruction, is a relatively less traumatic and highly safe procedure. It can lead to reconstruction of lumbosacral or lumbopelvic stability, while facilitating healing from TB-related inflammation.

\section{Abbreviations}

CT: Computed tomography; ESR: Erythrocyte sedimentation rate; JOA: Japanese Orthopaedic Association; MRI: Magnetic resonance imaging; ODI: Oswestry Disability index; TB: Tuberculosis; VAS: Visual analog scale

\section{Declarations}

\section{Acknowledgements}

Not applicable

\section{Authors' contributions}

Zhenchao Xu and Zhen Zhang participated in the design of this study, performed statistical analysis, and drafted the manuscript. Gang Chen collected the clinical data and follow-up details of the study and conducted the study. Xiyang Wang directed the study design and manuscript drafting. All authors read and approved the final manuscript.

\section{Funding}

This work was supported by the National Natural Science Foundation of China (No.81672191) and Xiangtan Science and Technology Project (SF-YB20181007). No benefit in any form has been or will be received from a commercial party related directly or indirectly to the subject of this manuscript.

\section{Availability of data and materials}

The datasets and materials generated or analyzed during the current study are available from the corresponding author on reasonable request.

\section{Ethics approval and consent to participate}

This study protocol was approved by the Ethics Committee of Xiangya Hospital and the written informed consent was obtained from all patients.

\section{Consent for publication}

All patients signed informed consent forms to publish their personal details in this article.

\section{Competing interests}

The authors declare that they have no competing interests 


\section{References}

1. World Health Organization. Global tuberculosis report 2019. Geneva: World Health Organization; 2019.

2. Hassan K, Elmorshidy E. Anterior versus posterior approach in surgical treatment of tuberculous spondylodiscitis of thoracic and lumbar spine. Eur Spine J. 2016;25(4):1056-63.

3. Jain AK, Kumar J. Tuberculosis of spine: neurological deficit. Eur Spine J. 2013;22(Suppl 4):624-33.

4. Wellons JC, Zomorodi AR, Villaviciencio AT, Woods CW, Lawson WT, Eastwood JD. Sacral tuberculosis: a case report and review of the literature. Surg Neurol. 2004;61(2):136-41.

5. Moon MS, Moon YW, Moon JL, Kim SS, Sun DH. Conservative treatment of tuberculosis of the lumbar and lumbosacral spine. Clin Orthop Relat Res. 2002; (398): 40-9.

6. Liu Z, Zhang P, Li W, Xu Z, Wang X. Posterior-only vs. combined posterior-anterior approaches in treating lumbar and lumbosacral spinal tuberculosis: a retrospective study with minimum 7-year follow-up. J Orthop Surg Res. 2020;15(1):99.

7. Zeng H, Wang X, Pang X, Luo C, Zhang P, Peng W, Wu P, Xu Z. Posterior only versus combined posterior and anterior approaches in surgical management of lumbosacral tuberculosis with paraspinal abscess in adults. Eur J Trauma Emerg Surg. 2014;40(5):607-16.

8. Zhang XF, Wang Y, Xiao SH, Liu ZS, Zhang YG, Liu BW, Xia ZM. Treatment of lumbar and lumbosacral spinal tuberculosis with minimally invasive surgery. Orthop Surg. 2010;2(1):64-70.

9. Kumar MN, Joseph B, Manur R. Isolated posterior instrumentation for selected cases of thoraco-lumbar spinal tuberculosis without anterior instrumentation and without anterior or posterior bone grafting. Eur Spine J. 2013;22(3):624-32.

10. Wang B, Kong L, Zhu Z, Gao W, Guo H, Wang X, Li H, Zhao Q, Zhang H, Hao D. Recurrent complex spinal tuberculosis accompanied by sinus tract formation: causes of recurrence and clinical treatments. Sci Rep. 2018;8(1):6933.

11. Zhang HQ, Lin MZ, Ge L, Li JS, Wu JH, Liu JY. Surgical management by one-stage posterior transforaminal lumbar debridement, interbody fusion, and posterior instrumentation for lumbo-sacral tuberculosis in the aged. Arch Orthop Trauma Surg. 2012;132(12):1677-83.

12. Lee CK, Vessa P, Lee JK. Chronic disabling low back pain syndrome caused by internal disc derangements. The results of disc excision and posterior lumbar interbody fusion. Spine. 1995;20(3):356-61.

13. Azimi P, Mohammadi HR, Montazeri A. An outcome measure of functionality and pain in patients with lumbar disc herniation: a validation study of the Japanese Orthopedic Association (JOA) score. J Orthop Sci. 2012;17(4):341-5.

14. van Hooff ML, Spruit M, Fairbank JC, van Limbeek J, Jacobs WC. The Oswestry Disability Index (version 2.1a): validation of a Dutch language version. Spine. 2015;40(2):E83-90.

15. Kirkaldy-Willis WH, Paine KW, Cauchoix J, Mclvor G. Lumbar spinal stenosis. Clin Orthop Relat Res. 1974; (99): 30-50.

16. Mori T, Leung CC. Tuberculosis in the global aging population. Infect Dis Clin North Am. 2010;24(3):751-68.

17. Xu Z, Wang X, Shen X, Luo C, Wu P, Zeng H. One-stage lumbopelvic fixation in the treatment of lumbosacral junction tuberculosis. Eur Spine J. 2015;24(8):1800-5.

18. Li W, Liu Z, Xiao X, Zhang Z, Wang X. Comparison of anterior transthoracic debridement and fusion with posterior transpedicular debridement and fusion in the treatment of mid-thoracic spinal tuberculosis in adults. BMC Musculoskelet Disord. 2019;20(1):570.

19. Bodapati PC, Vemula RCV, Mohammad AA, Mohan A. Outcome and management of spinal tuberculosis according to severity at a tertiary referral center. Asian J Neurosurg. 2017;12(3):441-6.

20. Ma YZ, Cui X, Li HW, Chen X, Cai XJ, Bai YB. Outcomes of anterior and posterior instrumentation under different surgical procedures for treating thoracic and lumbar spinal tuberculosis in adults. Int Orthop. 2012;36(2):299-305. 
21. Zaveri GR, Mehta SS. Surgical treatment of lumbar tuberculous spondylodiscitis by transforaminal lumbar interbody fusion (TLIF) and posterior instrumentation. J Spinal Disord Tech. 2009;22(4):257-62.

22. He Q, Xu J. Comparison between the antero-posterior and anterior approaches for treating L5-S1 vertebral tuberculosis. Int Orthop. 2012;36(2):345-51.

23. Benli IT, Kaya A, Acaroğlu E. Anterior instrumentation in tuberculous spondylitis: is it effective and safe? Clin Orthop Relat Res. 2007;460:108-16.

24. Jain AK, Dhammi IK, Prashad B, Sinha S, Mishra P. Simultaneous anterior decompression and posterior instrumentation of the tuberculous spine using an anterolateral extrapleural approach. J Bone Joint Surg Br. 2008;90(11):1477-81.

25. Tribus CB, Belanger T. The vascular anatomy anterior to the L5-S1 disk space. Spine. 2001;26(11):1205-8.

26. Li W, Liu J, Gong L, Zhou Y, Duan D. Posterior intervertebral space debridement, annular bone grafting and instrumentation for treatment of lumbosacral tuberculosis. BMC Surg. 2017;17(1):124.

27. Talu U, Gogus A, Ozturk C, Hamzaoglu A, Domanic U. The role of posterior instrumentation and fusion after anterior radical debridement and fusion in the surgical treatment of spinal tuberculosis: experience of 127 cases. J Spinal Disord Tech. 2006;19(8):554-9.

28. Huang QS, Zheng C, Hu Y, Yin X, Xu H, Zhang G, Wang Q. One-stage surgical management for children with spinal tuberculosis by anterior decompression and posterior instrumentation. Int Orthop. 2009;33(5):1385-90.

29. Bezer M, Kucukdurmaz F, Aydin N, Kocaoglu B, Guven O. Tuberculous spondylitis of the lumbosacral region: long-term follow-up of patients treated by chemotherapy, transpedicular drainage, posterior instrumentation, and fusion. J Spinal Disord Tech. 2005;18(5):425-9.

30. Wang YX, Zhang HQ, Liao W, Tang MX, Guo CF, Deng A, Wu JH, Liu JY. One-stage posterior focus debridement, interbody graft using titanium mesh cages, posterior instrumentation and fusion in the surgical treatment of lumbosacral spinal tuberculosis in the aged. Int Orthop. 2016;40(6):1117-24.

31. Vaccaro AR, Kim DH, Brodke DS, Harris M, Chapman JR, Schildhauer T, Routt ML, Sasso RC. Diagnosis and management of sacral spine fractures. Instr Course Lect. 2004;53:375-85.

32. Bellabarba C, Schildhauer TA, Vaccaro AR, Chapman JR. Complications associated with surgical stabilization of highgrade sacral fracture dislocations with spino-pelvic instability. Spine. 2006;31(11 Suppl):80-8.

33. Liu Z, Li W, Xu Z, Wang X, Zeng H. One-stage posterior debridement, bone grafting fusion, and mono-segment vs. shortsegment fixation for single-segment lumbar spinal tuberculosis: minimum 5-year follow-up outcomes. BMC Musculoskelet Disord. 2020;21(1):86.

34. Pang X, Wu P, Shen X, Li D, Luo C, Wang X. One-stage posterior transforaminal lumbar debridement, $360^{\circ}$ interbody fusion, and posterior instrumentation in treating lumbosacral spinal tuberculosis. Arch Orthop Trauma Surg. 2013;133(8):1033-9.

\section{Figures}



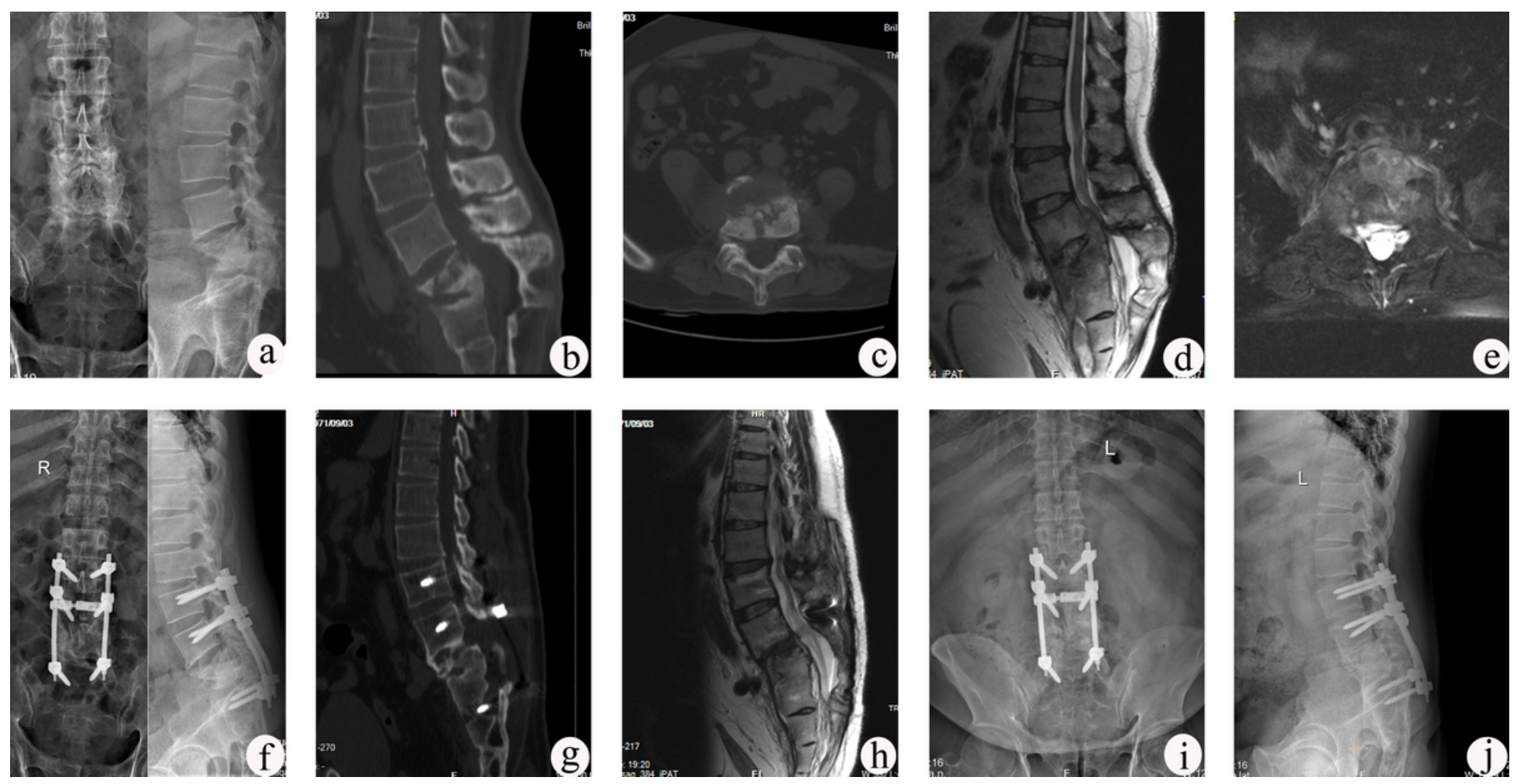

\section{Figure 1}

A 39-year-old male with L5/S1 TB underwent one-stage posterior debridement, interbody fusion, and lumbosacral fixation.

(a) Anterior and lateral radiographs of the lumbar spine showing the affected vertebrae with a lumbosacral angle of $22^{\circ}$. (b-

e) Preoperative CT and MRI demonstrating that the L5 vertebral body was severely damaged and collapsed, with cold abscess formation in the spinal canal. (f) Postoperative X-ray showing good internal fixation position with a lumbosacral angle of $28^{\circ}$. (g-h) Postoperative CT and MRI showing satisfactory bone fusion at 15 months and an unobstructed spinal canal. (i-j) X-ray displaying solid bone fusion and no displacement of instruments, with the correction loss of $1^{\circ}$ throughout 63 months of follow-up. Abbreviations: TB, tuberculosis; CT, computed tomography; MRI, magnetic resonance imaging.
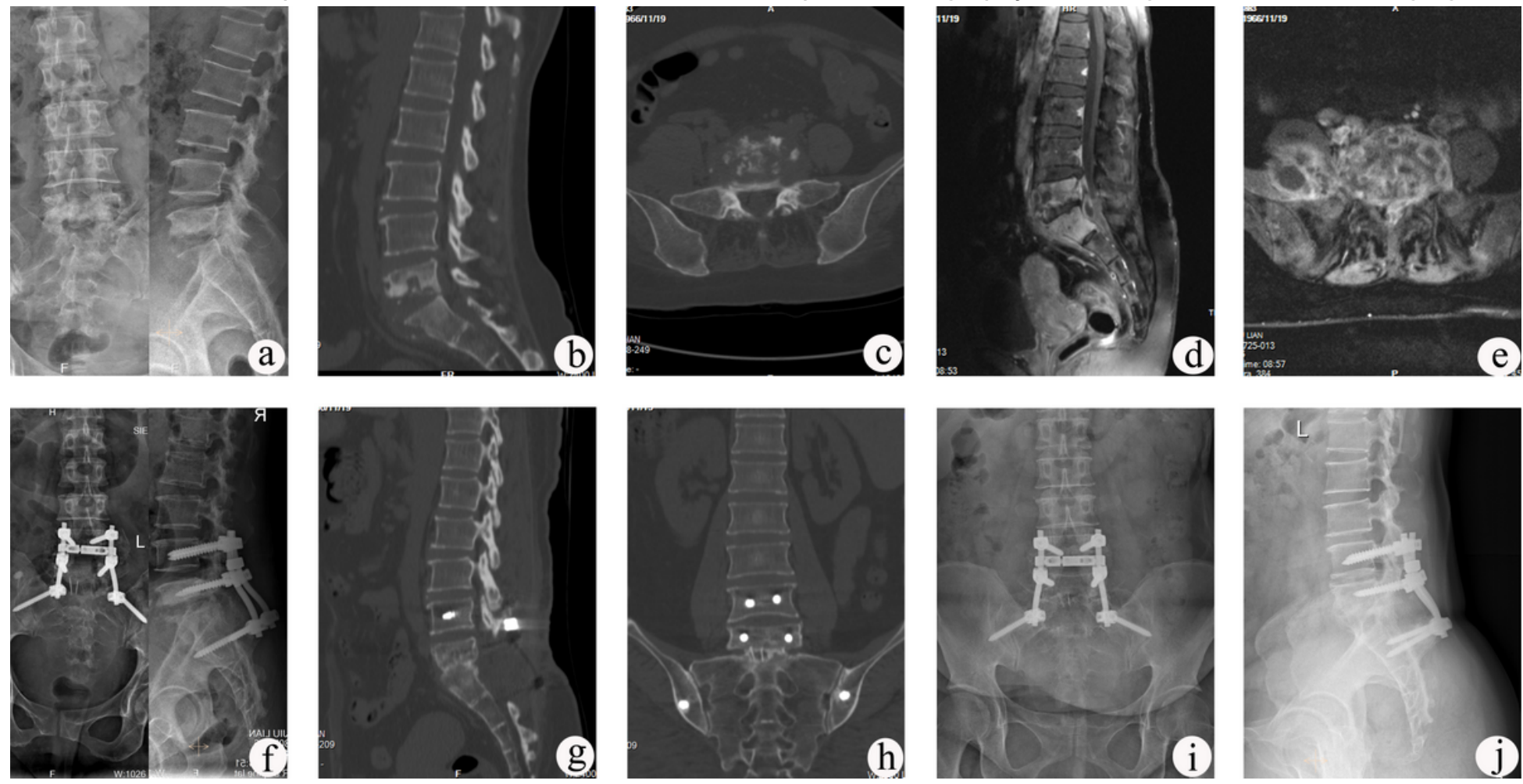


\section{Figure 2}

A 52-year-old female with L5/S1 TB underwent one-stage posterior debridement, interbody fusion, and lumbopelvic fixation. (a-e) Preoperative images showing that the TB lesion was located at L5/S1 and the lesion involved vertebral body destruction (the pedicle of S1 was also affected) and abscess formation. The lumbosacral angle was $23^{\circ}$. (f) Postoperative radiograph depicting correction of the deformity and the lumbosacral angle at $28^{\circ}$. (g-h) CT showing solid bone fusion was achieved at 15 months. (i-j) X-ray displaying the instruments in the correct a position, with a lumbosacral angle of $27^{\circ}$ at the follow-up period of 60 months. Abbreviations: TB, tuberculosis; CT, computed tomography.

\section{Supplementary Files}

This is a list of supplementary files associated with this preprint. Click to download.

- Data.docx 